\title{
The transcription factor OpWRKY2 positively regulates the biosynthesis of the anticancer drug camptothecin in Ophiorrhiza pumila
}

\author{
Xiaolong Hao', Chenhong Xie', Qingyan Ruan', Xichen Zhang², Chao Wu², Bing Han', Jun Qian', Wei Zhou', \\ Hans-Wilhelm Nützmann ${ }^{3}$ and Guoyin Kai (i) ${ }^{1}$
}

\begin{abstract}
The limited bioavailability of plant-derived natural products with anticancer activity poses major challenges to the pharmaceutical industry. An example of this is camptothecin, a monoterpene indole alkaloid with potent anticancer activity that is extracted at very low concentrations from woody plants. Recently, camptothecin biosynthesis has been shown to become biotechnologically amenable in hairy-root systems of the natural producer Ophiorrhiza pumila. Here, time-course expression and metabolite analyses were performed to identify novel transcriptional regulators of camptothecin biosynthesis in 0 . pumila. It is shown here that camptothecin production increased over cultivation time and that the expression pattern of the WRKY transcription factor encoding gene OpWRKY2 is closely correlated with camptothecin accumulation. Overexpression of OpWRKY2 led to a more than three-fold increase in camptothecin levels. Accordingly, silencing of OpWRKY2 correlated with decreased camptothecin levels in the plant. Further detailed molecular characterization by electrophoretic mobility shift, yeast one-hybrid and dualluciferase assays showed that OpWRKY2 directly binds and activates the central camptothecin pathway gene OpTDC. Taken together, the results of this study demonstrate that OpWRKY2 acts as a direct positive regulator of camptothecin biosynthesis. As such, a feasible strategy for the over-accumulation of camptothecin in a biotechnologically amenable system is presented.
\end{abstract}

\section{Introduction}

Camptothecin (CPT) is a monoterpenoid indole alkaloid (MIA) originally isolated from Chinese happy tree (Camptotheca acuminata $)^{1}$. This natural product exhibits potent antitumor activity by inhibiting DNA topoisomerase $\mathrm{I}^{1-3}$. Its two derivatives, namely, topotecan and irinotecan, have been approved by the Food and Drug Administration (FDA) of the United States for the treatment of various cancers ${ }^{4}$. Due to the wide range of targets of CPT derivatives, their clinical demand is continuously increasing.

Correspondence: Guoyin Kai (kaiguoyin@163.com)

'Laboratory of Medicinal Plant Biotechnology, College of Pharmacy, Zhejiang Chinese Medical University, 310053 Hangzhou, China

${ }^{2}$ Institute of Plant Biotechnology, School of Life Sciences, Shanghai Normal University, 200234 Shanghai, China

Full list of author information is available at the end of the article
Currently, commercial exploitation of camptothecin largely depends on extraction from natural woody plant resources such as C. acuminata and Nothapodytes foetida ${ }^{4}$. However, these plants typically show low camptothecin concentrations and are not suitable for modern biotechnological applications due to their long growth cycles. Several attempts have been made to establish robust plant cell culture systems to provide a platform for high CPT production. Among them are hairy-root cultures of Ophiorrhiza pumila, an herbal plant of the Ophiorrhiza genus in the Rubiaceae family. O. pumila produces camptothecin naturally in various organs, such as roots, stems, and leaves ${ }^{5}$. The $O$. pumila hairy-root system has been shown to produce up to $0.1-0.2 \%$ dry weight of camptothecin ${ }^{6-8}$. However, to further enhance camptothecin production in $O$. pumila by biotechnological engineering, it is crucial to

\section{(c) The Author(s) 2020}

(c) (i) Open Access This article is licensed under a Creative Commons Attribution 4.0 International License, which permits use, sharing, adaptation, distribution and reproduction cc) in any medium or format, as long as you give appropriate credit to the original author(s) and the source, provide a link to the Creative Commons license, and indicate if changes were made. The images or other third party material in this article are included in the article's Creative Commons license, unless indicated otherwise in a credit line to the material. If material is not included in the article's Creative Commons license and your intended use is not permitted by statutory regulation or exceeds the permitted use, you will need to obtain permission directly from the copyright holder. To view a copy of this license, visit http://creativecommons.org/licenses/by/4.0/. 


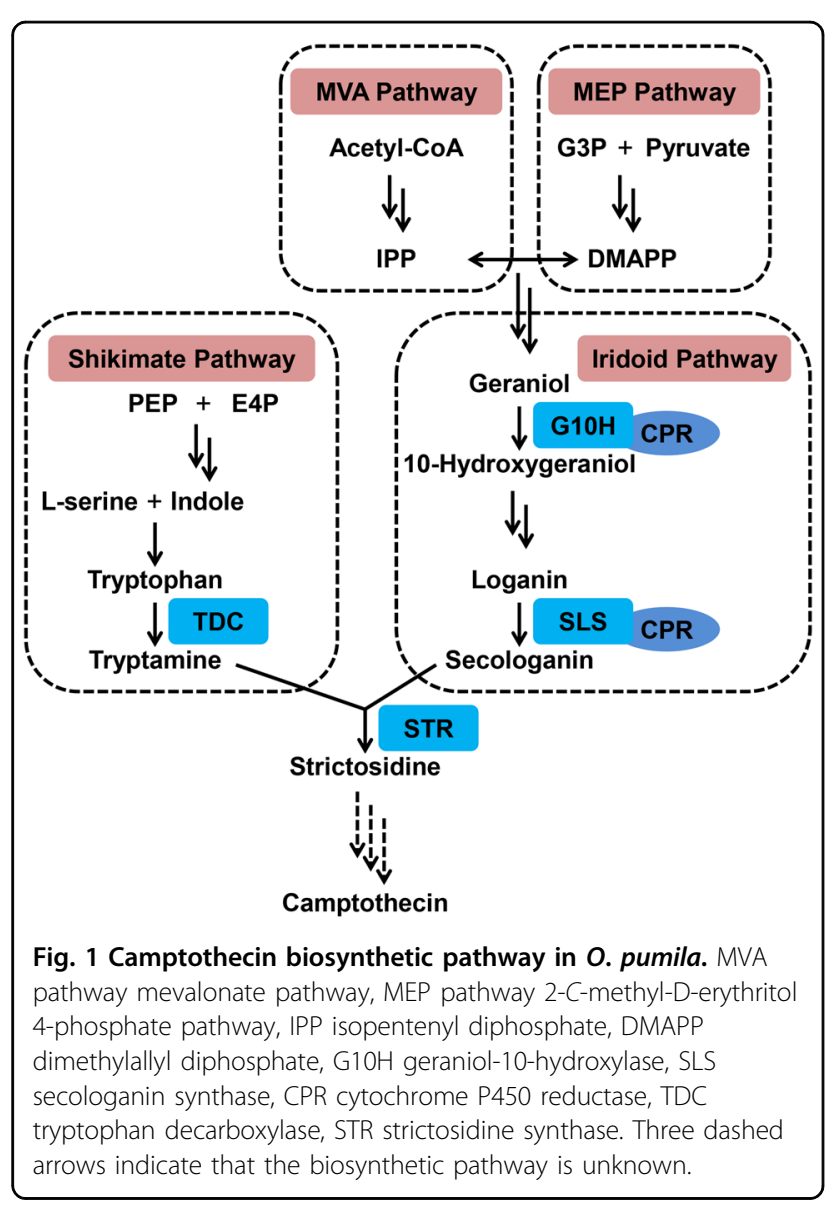

fully dissect its biosynthetic pathway and molecular regulatory mechanism.

The camptothecin biosynthesis pathway is complex and only partly resolved. Camptothecin biosynthesis uses intermediates of the iridoid and shikimate pathways, which converge to form the molecule strictosidine and then further, via unknown chemical reactions, form camptothecin (Fig. 1) $)^{4,9}$. Strictosidine synthase (OpSTR) catalyzes the condensation of tryptamine and secologanin to form strictosidine, a core intermediate in the camptothecin biosynthesis pathway ${ }^{10}$. Geraniol-10-hydroxylase $(\mathrm{OpG10H})$ and secologanin synthase (OpSLS), two cytochrome P450 (CYP450) family synthases, are involved in the conversion of geraniol to secologanin in the iridoid pathway $^{8}$. The cytochrome P450 reductase OpCPR is essential for the activity of OpG10H and OpSLS and plays an important role in electron transfer from nicotinamide adenine dinucleotide phosphate (NADPH) to cytochrome $\mathrm{P} 450^{8,10}$. Tryptophan decarboxylase (OpTDC) catalyzes the conversion of tryptophan to tryptamine in the shikimate pathway, ${ }^{5,10}$. Recent studies have confirmed that camptothecin biosynthesis can be manipulated by targeting these enzymes in metabolic engineering of $O$. pumila hairy roots. Suppression of $O p S L S$ and $O p T D C$ led to a decrease in camptothecin production in O. pumila hairy roots, thus corroborating their importance in camptothecin biosynthesis ${ }^{11}$. Manipulation of transgenic hairy-root systems further highlighted the important roles of both $O p G 10 H$ and $O p S L S$ in camptothecin biosynthesis, as co-overexpression of $\mathrm{OpG1OH}$ and OpSLS increased camptothecin levels in O. pumila hairy roots ${ }^{8}$. However, less is known about the molecular regulatory mechanism of camptothecin biosynthesis in O. pumila.

WRKY transcription factors (TFs) are one of the largest transcriptional regulator families in plants. They characteristically contain one or two WRKY domains. These domains consist of $\sim 60$ amino acid residues and enable DNA binding ${ }^{12-14}$. The WRKY domain contains a highly conserved WRKYGQK amino acid sequence at its $\mathrm{N}$ terminus and an atypical zinc-finger structure $\left(\mathrm{C}_{2} \mathrm{H}_{2}\right.$ or $\mathrm{C}_{2} \mathrm{HC}$ ) at its C-terminus ${ }^{12,13,15}$. In plants, WRKY TFs are divided into three groups based on the number of WRKY domains (two WRKY domains in Group I and one in Group II and III) and the structure of the zinc fingers $\left(\mathrm{C}_{2} \mathrm{H}_{2}\right.$ in Group I and II and $\mathrm{C}_{2} \mathrm{HC}$ in Group III proteins) ${ }^{12,13}$. The WRKY domains generally recognize and bind to W-box (TTGACT/C) DNA motifs at target regions. Previous studies have shown that WRKY proteins, especially WRKY III subgroup members, are involved in the regulation of biosynthesis and accumulation of plant secondary metabolites in medicinal plants. For example, CrWRKY1, a WRKY III subgroup TF, has a positive regulatory effect on the biosynthesis of monoterpenoid indole alkaloids by binding and activating CrTDC in Catharanthus roseus ${ }^{16}$. In Artemisia annua, the WRKY III subgroup protein AaWRKY1 was reported to positively regulate artemisinin biosynthesis by promoting the transcription of AaADS, AaCYP71AV1 and $A a D B R 2^{17}$. In Salvia miltiorrhiza, the WRKY III subgroup member SmWRKY1 was shown to positively regulate tanshinone biosynthesis by activating the $\operatorname{SmDXR}$ gene $^{15}$. WsWRKY1, a WRKY III subgroup TF in Withania somnifera, was shown to positively regulate the biosynthesis of triterpenoids by activating WSSQS and $W S S Q E^{18}$. Recently, two WRKY III subgroup TFs, namely, OpWRKY1 and OpWRKY3, have been implicated in camptothecin biosynthesis in O. pumila hairy roots ${ }^{5,19}$. OpWRKY1 negatively regulates camptothecin biosynthesis, and OpWRKY3 plays a minor regulatory role in camptothecin production by affecting the development of $O$. pumila hairy roots and activating the expression of $O p C P R^{5,19}$. In addition, several TFs from other families have also been reported to be involved in the regulation of camptothecin biosynthesis. OpERF2, an ERF TF family protein, has been isolated and shown to play a positive role in regulating camptothecin biosynthesis ${ }^{20}$. Moreover, introduction of the MYB TF member OpMYB1 into $O$. pumila hairy roots reduced camptothecin production ${ }^{21}$. 
In C. acuminata, a bZIP transcription factor, CaLMF, is a significant light signaling component and mediates lightregulated camptothecin biosynthesis ${ }^{22}$. In general, transcriptional regulation plays an important role in the biosynthesis of bioactive metabolites in medicinal plants.

In this study, the biosynthesis of camptothecin in $O$. pumila hairy roots at different growth stages was systematically investigated. By analyzing the expression of all putative WRKY TFs of $O$. pumila at different growth stages, a single transcription factor, $O p W R K Y 2$, was identified as being highly coexpressed with known camptothecin biosynthesis pathway genes. Overexpression of OpWRKY2 in O. pumila hairy roots resulted in increased levels of OpTDC expression as well as camptothecin and tryptamine formation. Accordingly, downregulation of $O p W R K Y 2$ reduced $O p T D C$ expression levels and camptothecin production. Biochemical characterization showed that OpWRKY2 binds and activates the promoter of $O p T D C$ in vitro and in vivo. Altogether, our analyses identified a novel regulator of camptothecin biosynthesis and a potential target for advanced bioengineering for enhanced production of an important anticancer drug.

\section{Materials and methods \\ Plant materials}

In this study, the O. pumila plants originated from Fujian Province of China and were cultivated in a greenhouse at Zhejiang Chinese Medical University. The O. pumila plantlets used for analysis of tissue expression patterns and hairy-root transformation were obtained and cultured in solid B5 medium as previously reported ${ }^{7}$. Three different tissues of two-month-old O. pumila sterile plants (roots, stems and leaves) were separately collected for tissue expression pattern analyses of OpWRKY2 and $O p T D C$ and camptothecin content detection in different tissues. Different $O$. pumila hairy-root lines were inoculated and cultured in liquid B5 medium at $120 \mathrm{rpm}$ in darkness at $25^{\circ} \mathrm{C}$. Nicotiana benthamiana plants used for subcellular localization analyses of OpWRKY2 protein and dual-luciferase (Dual-LUC) assays to detect the activation of OpWRKY2 were grown in pots containing soil mixture and placed in a growth chamber under a light/ dark photoperiod of $16 / 8 \mathrm{~h}$ at $25^{\circ} \mathrm{C}^{23}$.

\section{Hairy roots at different growth stages and phytohormone treatments}

For analysis of growth phenotype and camptothecin production in O. pumila hairy roots, $0.2 \mathrm{~g}$ of fresh $\mathrm{C} 58 \mathrm{C} 1$ hairy-root lines (infected with modified Agrobacterium tumefaciens strain $\mathrm{C} 58 \mathrm{C} 1$ ) with uniform growth were inoculated in B5 liquid medium and cultured for 60 days $^{7,15}$. Hairy roots and culture medium were harvested at $10,20,30,40,50$, and 60 days and used to detect gene expression and metabolite levels. For the analysis of $O p W R K Y 2$ gene expression in response to plant hormones, $\mathrm{C} 58 \mathrm{C} 1$ hairy-root lines were cultured for 30 days and subsequently treated with $50 \mu \mathrm{M}$ salicylic acid (SA), $50 \mu \mathrm{M}$ gibberellin $\left(\mathrm{GA}_{3}\right), 100 \mu \mathrm{M}$ abscisic acid (ABA), and $200 \mu \mathrm{M}$ methyl jasmonate (MeJA) $)^{5}$. An equivalent volume of ethanol was used as a control. The hairy roots were harvested at $0,0.5,1,3,6,9$, and $12 \mathrm{~h}$ post hormone application and immediately frozen in liquid nitrogen for further quantitative real-time polymerase chain reaction (qRT-PCR) analyses.

\section{Plant RNA extraction and qRT-PCR analyses}

Total RNA of all samples was extracted using the Plant RNAprep Pure Kit (Tiangen, Beijing, China). cDNA synthesis from total RNA and qRT-PCR analyses of all gene transcripts were performed as previously described $^{24}$. The housekeeping gene OpActin in O. pumila was used as an internal control gene in qRT-PCR for normalization of all samples. All gene-specific primer sequences of all camptothecin biosynthesis pathway genes and $O p W R K Y$ genes used for qRT-PCR analyses are listed in Table S1. The relative gene expression values in all samples were calculated using the $2^{-\Delta \Delta \mathrm{Ct}}$ method. All qRT-PCR analyses of each sample were performed for three biological replicates.

\section{Bioinformatics analysis}

The bioinformatics analyses were performed as previously described ${ }^{25}$. WRKY homologues were identified in the $O$. pumila root and hairy-root transcriptome sequences generated by our laboratory using the hidden Markov model (HMM) search and BLASTx program with an Evalue $<10^{-5} 8,25$. Redundant sequences were manually removed. All OpWRKY proteins in O. pumila were aligned using the ClustalW program with the default parameters ${ }^{26}$. The phylogenetic tree of all OpWRKY proteins in $O$. pumila was constructed and performed using the neighbor-joining method with MEGA5 software ${ }^{27}$. The bootstrap values were calculated from 1000 replicates to analyze and assess the accuracy of the phylogeny.

\section{Subcellular localization}

To analyze the subcellular localization of the OpWRKY2 protein, the open reading frame (ORF) was amplified by PCR from the O. pumila hairy-root cDNA library with $O p W R K Y 2$ gene-specific primers (Table S2) and inserted into the modified plant expression vector $p H B-Y F P$ (yellow fluorescent protein) to generate the $p H B-O p W R K Y 2-Y F P$ construct. The $p H B-Y F P$ construct without $O p W R K Y 2$ was used as the negative control. The plasmids $p H B$ $O p W R K Y 2-Y F P$ and $p H B-Y F P$ were introduced into the A. tumefaciens strain GV3101 and transiently infected the epidermal cells of 5-week-old $N$. benthamiana leaves. YFP 
signals were analyzed using an LSM880 confocal laser microscope (Carl Zeiss, Germany) $48 \mathrm{~h}$ post infection, and three biological replicates were performed to confirm the results as reported previously ${ }^{23,28}$.

Generation of OpWRKY2 transgenic $O$. pumila hairy roots

The full-length $O p W R K Y 2$ ORF fragment was amplified with Spe I and BstE II restriction sites and inserted into the previously modified $p C A M B I A 2300^{+}$plant expression vector to obtain the $p C A M B I A 2300^{+}-O p W R K Y 2$ construct, which was used for overexpression of OpWRKY2 in O. pumila hairy roots (Fig. S1a $)^{7,15}$. Chimeric repressor silencing by using the EAR motif repression domain (SRDX) is a very common technique that is widely used to study the function of transcription factors ${ }^{29}$. It is very useful not only for the rapid analysis of the function of redundant transcription factors but also for the manipulation of biological traits via the suppression of gene expression that is regulated by specific transcription factors $^{29}$. In this study, to further examine the function of $O p W R K Y 2$, the DNA sequence encoding the SRDX repressor domain (LDLDLELRLGFA) was fused to the Cterminus of OpWRKY2 and inserted into the modified $p C A M B I A 2300^{+}$to generate the $p C A M B I A 2300^{+}$OpWRKY2-SRDX construct (Fig. S1b) ${ }^{16,29}$. The $p C A M-$ $B I A 2300^{+}$empty vector without $O p W R K Y 2$ was used as the control. All plasmids were transferred into the disarmed A. tumefaciens strain $\mathrm{C} 58 \mathrm{C} 1$, harboring the Agrobacterium rhizogenes Ri plasmid pRiA4, and subsequently transformed into O. pumila stems to generate O. pumila transgenic hairy-root lines ${ }^{7,15}$. The screening of transformed hairy-root cultures was carried out on B5 medium plates. The positive transgenic hairy-root lines (OpWRKY2-OE and OpWRKY2-SRDX) were verified via PCR amplification of hairy-root genomic DNA. Primers used for PCR amplification of positive transgenic hairy roots were designed to cover the $O p W R K Y 2$ gene and partial $p C A M B I A 2300^{+}$vector sequences and are listed in Table S2. Positive transgenic hairy roots were further inoculated and cultured in B5 liquid medium for 45 days in the dark. Harvested hairy-root lines were used for gene expression and metabolite analysis.

\section{Determination of metabolites in hairy roots by HPLC}

For a time series of metabolite production in O. pumila, hairy roots were harvested every 10 days for 60 days. Hairy roots of transgenic lines, namely, OpWRKY2-OE and $O p W R K Y 2-S R D X$, were harvested after 45 days of cultivation. All samples were dried at $50{ }^{\circ} \mathrm{C}$ and thoroughly ground, and the liquid medium was directly evaporated. These samples were used for further metabolite measurements.

For camptothecin and tryptamine measurements, $\sim 0.1 \mathrm{~g}$ of dried hairy-root powder was extracted with
$20 \mathrm{~mL}$ of methanol and sonicated for $1 \mathrm{~h}$. After centrifugation, the supernatant was evaporated under vacuum, and the residual material was dissolved in $2.0 \mathrm{~mL}$ of methanol and filtered through a $0.22 \mu \mathrm{m}$ filter. High-performance liquid chromatography (HPLC) analysis of camptothecin and tryptamine extracts was performed using an Agilent 1260 detector equipped with a reversed-phase C18 column (Agilent Technologies, Palo Alto, CA, USA) as previously described ${ }^{5,8}$. The camptothecin detection conditions were as follows: mobile phase, acetonitrile:water $(65: 35, \mathrm{v} / \mathrm{v})$; column temperature, $30^{\circ} \mathrm{C}$; and detection wavelength, $254 \mathrm{~nm}$. Tryptamine detection conditions were as follows: mobile phase, $43 \%$ acetonitrile: $30 \%$ methanol:26\% double distilled water:1\% glacial acetic acid (v/v); column temperature, $30{ }^{\circ} \mathrm{C}$; detection wavelength, $254 \mathrm{~nm}$. Commercially available standards of camptothecin and tryptamine (Aladdin, Shanghai, China) were used for identification and comparative quantification as previously described ${ }^{5,8}$.

For loganin and secologanin measurements, $\sim 0.1 \mathrm{~g}$ of dried hairy-root powder was extracted with $10 \mathrm{~mL}$ of ethanol:water (4:1, v/v) and sonicated for $30 \mathrm{~min}$. After centrifugation, the supernatant was evaporated under vacuum, and the residual material was dissolved in $2.0 \mathrm{~mL}$ of water and filtered through a $0.22 \mu \mathrm{m}$ filter. HPLC analysis of loganin and secologanin extracts was performed using an Agilent 1260 detector equipped with a reversed-phase C18 column (Agilent Technologies, Palo Alto, CA, USA) as previously described ${ }^{5,8}$. The detection conditions of loganin and secologanin were as follows: mobile phase, acetonitrile:water (25:75, $\mathrm{v} / \mathrm{v}$ ); column temperature, $30^{\circ} \mathrm{C}$; and detection wavelength, $236 \mathrm{~nm}$. Commercially available standards of loganin and secologanin (Aladdin, Shanghai, China) were used for comparative quantification as previously described $^{5,8}$.

\section{Recombinant protein production and EMSA}

To express and purify the recombinant protein, the full-length $O p W R K Y 2$ ORF fragment was cloned into the BamH I and Hind III sites of the $p$ Cold-TF vector. The constructs were verified by DNA sequencing and transformed into Escherichia coli strain Rosetta (DE3) cells to produce His-tagged fusion proteins. The $p$ ColdTF empty vector without $O p W R K Y 2$ was used as the negative control. Transformed Rosetta cell cultures used for the expression of HIS recombinant protein were induced by adding isopropyl $\beta$-D-thiogalactopyranoside (IPTG) at a final concentration of $0.1 \mathrm{mM}$ at an optical density of approximately 0.6 at $600 \mathrm{~nm}$. After induction for $14 \mathrm{~h}$ at $16^{\circ} \mathrm{C}$, Rosetta cells were harvested by centrifugation and purified using Ni-NTA (nitrilotriacetic acid) agarose (Invitrogen, USA) as previously 
described ${ }^{16}$. To investigate the ability of the OpWRKY2 protein to bind to the $\mathrm{W}$-box in the $O p T D C$ promoter, the $3090 \mathrm{bp}$ upstream region of the OpTDC gene was analyzed. For Electrophoretic mobility shift assay (EMSA) experiments, DNA probes were designed based on the native $O p T D C$ promoter sequence $(-2565$ to -2552 relative to the ATG) containing a single W-box. Complementary oligonucleotides labeled with biotin at the 5' end of each strand were synthesized and annealed to produce double-stranded probes for EMSA. EMSAs were performed as previously described ${ }^{16}$.

\section{Dual-LUC assays}

To investigate the ability of OpWRKY2 to transcriptionally activate the OpTDC gene, the $3090 \mathrm{bp}$ promoter of $O P T D C$ was analyzed and cloned into the $p$ GreenII0800-LUC vector. The reporter constructs $w t-p O p T D C:: f L U C$ and mutant-pOpTDC::fLUC were obtained by inserting the native promoter of $O p T D C$ and a mutated version into the pGreenII0800-LUC vector to drive expression of the firefly luciferase gene $^{30}$. The Renilla luciferase gene driven by the CaMV $35 \mathrm{~S}$ promoter was used as an internal control. The assembled vectors were cotransformed with the helper plasmid pSoup19 into A. tumefaciens strain GV3101. The A. tumefaciens strain GV3101 containing $p H B$ $O p W R K Y 2-Y F P$ was used as the effector, and $p H B-Y F P$ was used as the negative control. Infiltration and detection were performed as previously described, with minor modifications ${ }^{31}$. The reporter strains harboring $w t-p O p T D C: \because f L U C$ or mutant-pOpTDC::fLUC were mixed with effector strains harboring either $p H B$ $O p W R K Y 2-Y F P$ or $p H B-Y F P$ at a ratio of 1:1. Leaves were collected after $48 \mathrm{~h}$, and Dual-LUC assays were performed using the Dual-Luciferase Reporter Assay System according to the manufacturer's instructions (Promega, Madison, WI, USA). Three biological replicates per treatment were measured. All primers used to amplify the $O p T D C$ promoter are listed in Table S2.

\section{$\mathrm{Y} 1 \mathrm{H}$ assays}

For $\mathrm{Y} 1 \mathrm{H}$ assays, the full-length $O p W R K Y 2$ ORF fragment was amplified and cloned into the effector plasmid $p B 42 A D$. The triple tandem copy of the $p O p T D C \mathrm{wt}-\mathrm{W}-$ box motif (CTTCAGTCAAGGCC) and mutant-W-box motif (CTTCAttttAGGCC) were inserted into the reporter plasmid pLacZ between EcoR I and Xho I. Y1H assays were performed as previously described ${ }^{23,32}$. Effector and reporter plasmids were cotransformed into yeast strain EGY48a. Transformants were cultivated on SD/-Ura/-Trp medium for $48 \mathrm{~h}$ and tested on SD/-Ura/-Trp medium with 5-bromo-4-chloro-3-indolyl- $\beta$-D-galactopyranoside (X-gal) for $24 \mathrm{~h}$. Empty $p B 42 A D$ and $p L a c Z$ plasmids were cotransformed into yeast and used as negative controls. All primers used to amplify OpWRKY2 and DNA motifs are listed in Table S2.

\section{Statistical analyses}

All experiments in this study were conducted with at least three biological replicates. All data are presented as the mean \pm standard deviation (SD). To test the statistically significant differences between the control and treated samples/transgenic hairy-root lines, a paired twotailed Student's $t$-test was conducted with a significance threshold of $p<0.05$.

\section{Results}

Camptothecin biosynthesis at different growth stages of $O$. pumila hairy roots

Hairy roots of O. pumila have the potential to synthesize camptothecin, yet the association of cultivation time and camptothecin production remains poorly characterized. To investigate the capacity of $O$. pumila hairy roots to produce camptothecin at different growth stages, $\sim 0.2 \mathrm{~g}$ of fresh hairy roots with uniform growth were inoculated in B5 liquid medium and cultured for 60 days. As shown in Fig. 2a, b, the color and biomass of the hairy roots changed at different growth stages over time. The color of the hairy roots gradually intensified, changing from yellow to dark yellow, and the color of the medium changed from colorless to yellow (Fig. 2a). The biomass of the hairy roots gradually increased, reaching a maximum of $0.89 \mathrm{~g}$ dry weight at 40 days (Fig. 2b).

Since camptothecin in O. pumila hairy roots could be excreted into the culture medium, we analyzed hairy roots and liquid medium separately by HPLC analysis to monitor the accumulation of camptothecin in O. pumila (Fig. 2c). The total yield of camptothecin increased over time and reached a maximum of $2.48 \mathrm{mg} /$ flask at 50 days (Fig. 2c). Interestingly, the content of camptothecin in the culture medium increased throughout the growth period and reached $0.87 \mathrm{mg} /$ flask at 60 days. In contrast, the content of camptothecin extracted from hairy roots peaked at 40 days at $\sim 1.93 \mathrm{mg} /$ flask (Fig. 2c).

In parallel to the metabolite analyses, the expression levels of camptothecin biosynthetic genes $(O p G 10 H$, $O p S L S, O p C P R, O p T D C$, and $O p S T R$ ) were determined by qRT-PCR analyses. As shown in Fig. 2d, the relative transcript levels of all camptothecin biosynthetic genes increased over time. The highest upregulation was detected for the biosynthesis genes $O p T D C$ and $O p S T R$, reaching maximum expression levels after 40 days (Fig. 2d). After 50 days, the relative expression levels of $O p G 10 H, O p S L S$, $O p T D C$, and OpSTR decreased (Fig. 2d). Expression of the $O P C P R$ gene peaked at 50 days and decreased slightly at the final time point (Fig. 2d). Altogether, these data suggested that camptothecin biosynthesis in O. pumila hairy roots changes dynamically over time. 


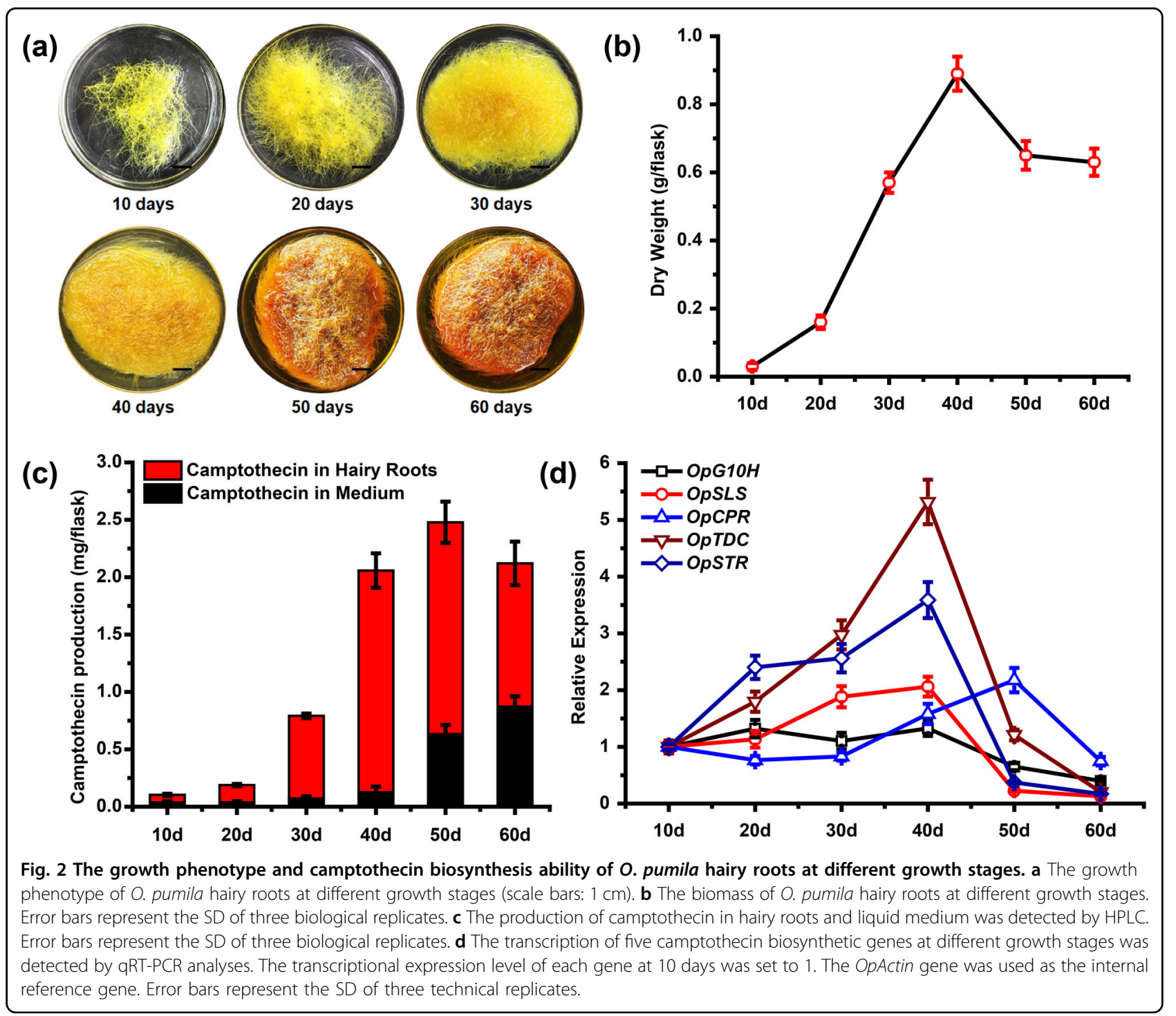

\section{Identification of expressed WRKY TFs in O. pumila hairy roots}

Previously, it has been reported that WRKY TFs are involved in the regulation of secondary metabolism in medicinal plants. To systematically investigate the importance of OpWRKYs in the positive regulation of camptothecin biosynthesis, an available $O$. pumila hairy-root transcriptome dataset was analyzed for expressed WRKY domain-encoding genes. Thirty-four putative WRKY transcription factor genes encoding either one or two WRKY domains were identified (Fig. 3a, b). Phylogenetic analysis based on the protein sequences divided the putative OpWRKY TFs into three groups (Fig. 3a, b). Protein alignments showed that 8 OpWRKYs fell into WRKY TF group I, 22 OpWRKYs to WRKY TF group II, and 4 OpWRKYs to WRKY TF group III (Fig. 3a).
To further characterize these WRKY TFs and to better understand their role in camptothecin biosynthesis, the relative expression levels of all putative WRKY TFs were determined by qRT-PCR at different growth stages by qRT-PCR (Fig. 3c). Variable expression trends were observed for the OpWRKY TF genes. The expression levels of a number of OpWRKY genes increased over time, while the expression levels of others gradually decreased. The expression pattern of only one OpWRKY TF gene, OpWRKY2, closely mirrored the pattern observed for camptothecin biosynthetic genes. OpWRKY2 expression gradually increased until it reached a peak at 40 days, slightly decreased at 50 days and dropped to a very low level at 60 days (Fig. 3c). Taken together, these results indicated that OpWRKY2 might be a candidate gene involved in the positive regulation of camptothecin biosynthesis. 

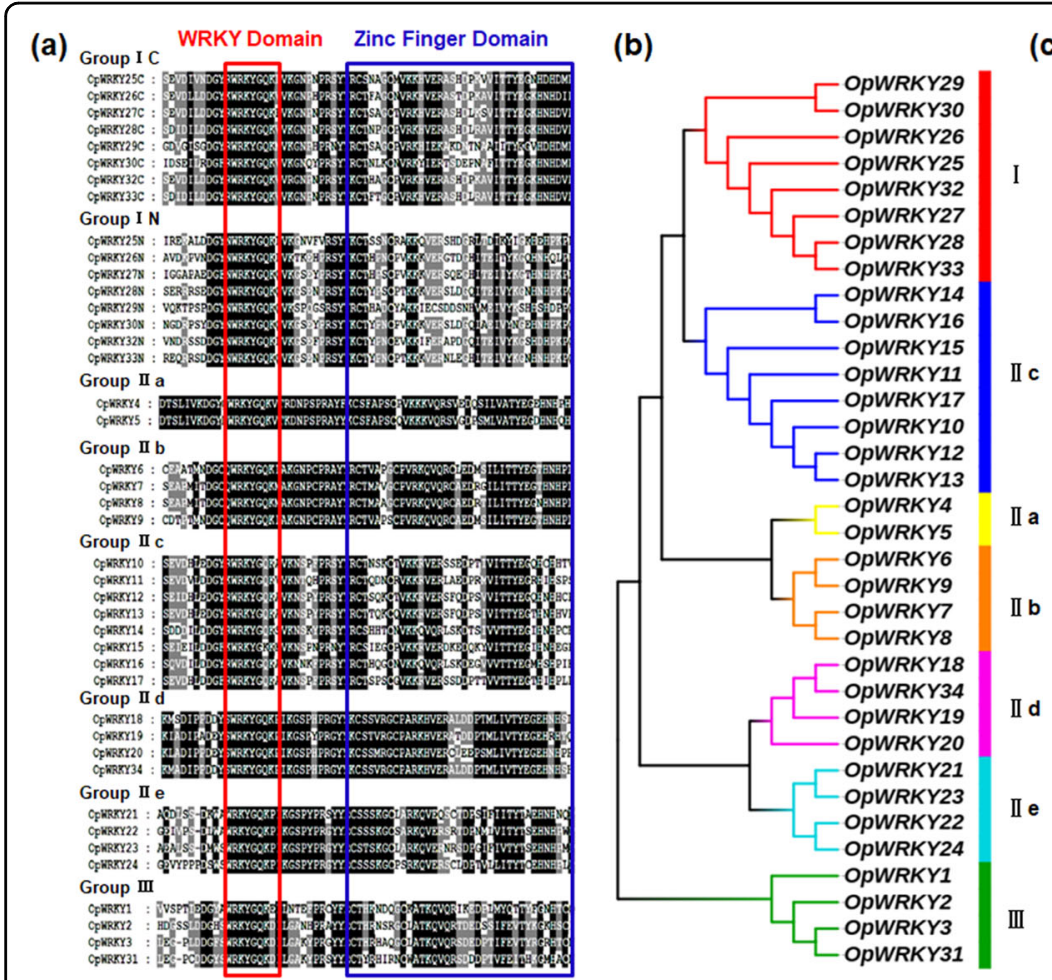

(c)
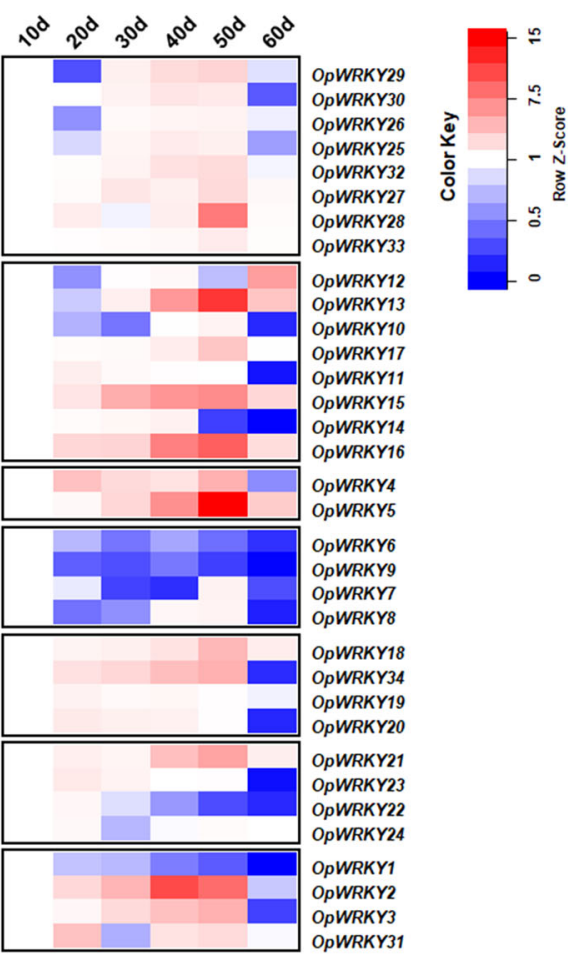

Fig. 3 Sequence and expression analysis of OpWRKYs. a Protein sequence alignment of OpWRKYs. The conserved WRKY domains are highlighted in red; the conserved zinc-finger domains are highlighted in blue. b Phylogenetic analysis of OpWRKYs. c The expression levels of all OpWRKY genes at different growth stages were detected by qRT-PCR analyses. The expression level of each gene at 10 days was set to 1 . The OpActin gene was used as the internal reference gene.

\section{Expression profiling and subcellular localization of OpWRKY2}

To further investigate the expression pattern of $O p W R K Y 2$, we collected materials from three different tissues (root, stem and leaf) of 2-month-old O. pumila plants and analyzed camptothecin biosynthesis and the relative transcript levels of $O p W R K Y 2$. First, we investigated the content of camptothecin in different tissues and found that the accumulation level of camptothecin in the roots, stems and leaves of $O$. pumila was quite high, with the highest content observed in roots (Fig. 4a). Then, we detected the expression of the camptothecin biosynthetic gene $O p T D C$ in different tissues and found that it was expressed in roots, stems and leaves, and the expression level was relatively high in roots (Fig. 4b). In addition, the highest transcript levels of OpWRKY2 were detected in leaves, intermediate levels in roots and low levels in stems (Fig. 4c). These results indicate that OpWRKY2 might not only be involved in the regulation of camptothecin biosynthesis but also regulate other processes in plant growth and development.

Next, the transcriptional response of OpWRKY2 to exogenous phytohormone treatments was analyzed. Thirtyday-old $O$. pumila hairy roots were individually treated with the plant hormones $\mathrm{SA}, \mathrm{GA}_{3}, \mathrm{ABA}$ and MeJA, and samples were taken after $0,0.5,1,3,6,9$, and $12 \mathrm{~h}$. The results presented in Fig. 4d show that application of each hormone led to rapid and significant upregulation of $O p W R K Y 2$ transcript levels (Fig. 4d). In response to SA and ABA, OpWRKY2 expression increased 14-fold after $1 \mathrm{~h}$ and was maintained at high levels for at least $3 \mathrm{~h}$. In contrast, in response to GA and MeJA, OpWRKY2 expression peaked within $1 \mathrm{~h}$ and returned to near basal levels at $3 \mathrm{~h}$. These results indicate an important role of hormones in the transcriptional regulation of OpWRKY2.

To analyze the subcellular localization of OpWRKY2, we carried out transient transformation assays in $N$. benthamiana leaves. As shown in Fig. 4e, fluorescent $O p W R K Y 2$-reporter signals were specifically detected in the nucleus of $N$. benthamiana leaf cells, while the YFP control protein was distributed throughout the cells (Fig. 4e). This nuclear localization is in accordance with the expected role of OpWRKY2 as a transcription factor.

\section{Generation of the OpWRKY2-OE and OpWRKY2-SRDX hairy- root lines}

To establish a role for $O p W R K Y 2$ in camptothecin biosynthesis, OpWRKY2 overexpression and silencing lines were generated. For $O$. pumila hairy roots, the recombinant overexpression and silencing constructs were 


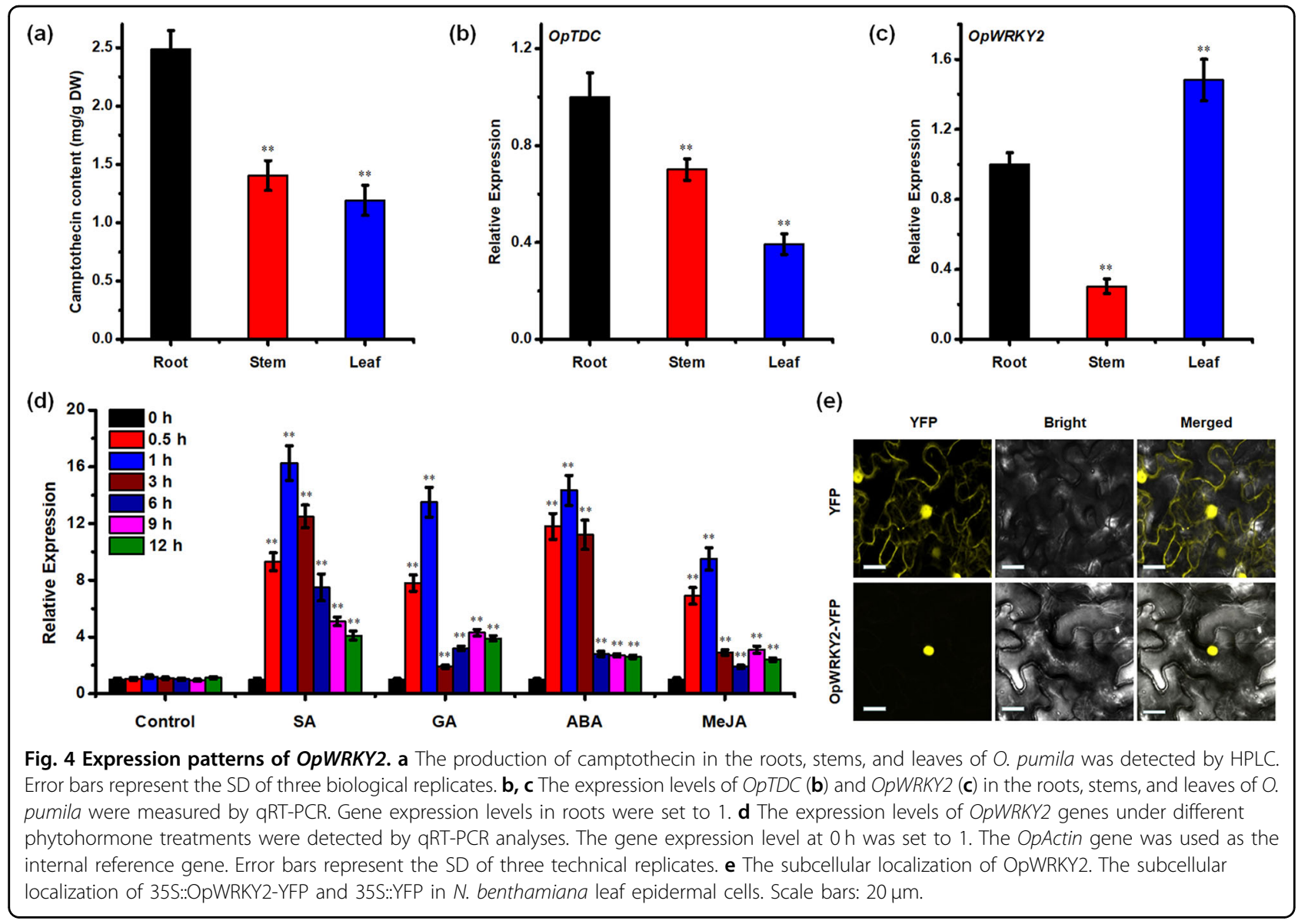

introduced to O. pumila explant stems by Agrobacteriummediated transformation (Fig. 5a-e). Positive transgenic hairy-root lines carrying $O p W R K Y 2$ were identified by PCR on genomic DNA of $O$. pumila using gene-specific primers (Table S2). The results showed that 17 out of 48 tested $O P W R K Y 2-O E$ hairy roots were successfully transformed (35.4\% positive rate) (Fig. S2). In addition, 15 out of 32 candidate $O p W R K Y 2-S R D X$ hairy roots were successfully transformed (46.9\% positive rate) (Fig. S3). Expression analysis of OpWRKY2 in OpWRKY2-OE lines showed a 1.03 - to 36.55 -fold increase in relative transcript levels compared to the wild-type control (Fig. 5f). In OpWRKY2$S R D X$ hairy-root lines, relative transcript levels of $O p W R K Y 2$ were increased 1.26- to 25.12-fold compared to the wild-type control (Fig. 5g). For both OpWRKY2-OE and $O P W R K Y 2-S R D X, 4$ lines with the highest increase in expression were selected for further analysis.

\section{Expression analysis of camptothecin biosynthesis genes in OpWRKY2-OE and OpWRKY2-SRDX hairy roots}

To analyze the impact of $O p W R K Y 2$ on the expression of camptothecin pathway genes, the relative transcript levels of $O p G 10 H, O p S L S, O p C P R, O p T D C$, and $O p S T R$ were measured in $O p W R K Y 2$ overexpression and silencing lines
(Fig. 6a). The relative transcript levels of $O p T D C$ were increased almost 10-fold in OpWRKY2-OE hairy-root lines compared to the control. Accordingly, OpTDC expression levels were significantly decreased in the OpWRKY2-SRDX hairy-root lines, suggesting a positive regulatory role of $O p W R K Y 2$ in $O p T D C$ expression. The relative expression of $O p G 10 H$ and $O p S L S$, two CYP450 family genes in the iridoid pathway, did not change significantly in the $O P W R K Y 2-O E$ and OpWRKY2-SRDX hairy-root lines. In addition, the expression levels of $O p C P R$ and $O p S T R$ were increased slightly in the OpWRKY2-OE hairy-root lines compared to the control, but there was no obvious change in the OpWRKY2-SRDX hairy-root lines. In conclusion, OpWRKY2 positively regulates the expression of OpTDC in $O$. pumila hairy roots.

\section{Metabolite analysis of OpWRKY2-OE and OpWRKY2-SRDX hairy roots}

To analyze metabolite levels in the generated lines, hairy roots of O. pumila were inoculated into $100 \mathrm{~mL}$ of liquid B5 medium for 45 days and then collected for further study. Neither overexpression nor silencing of $O p W R K Y 2$ significantly affected the dry weight of the analyzed hairy roots compared to the wild-type control (Fig. 6b). HPLC 


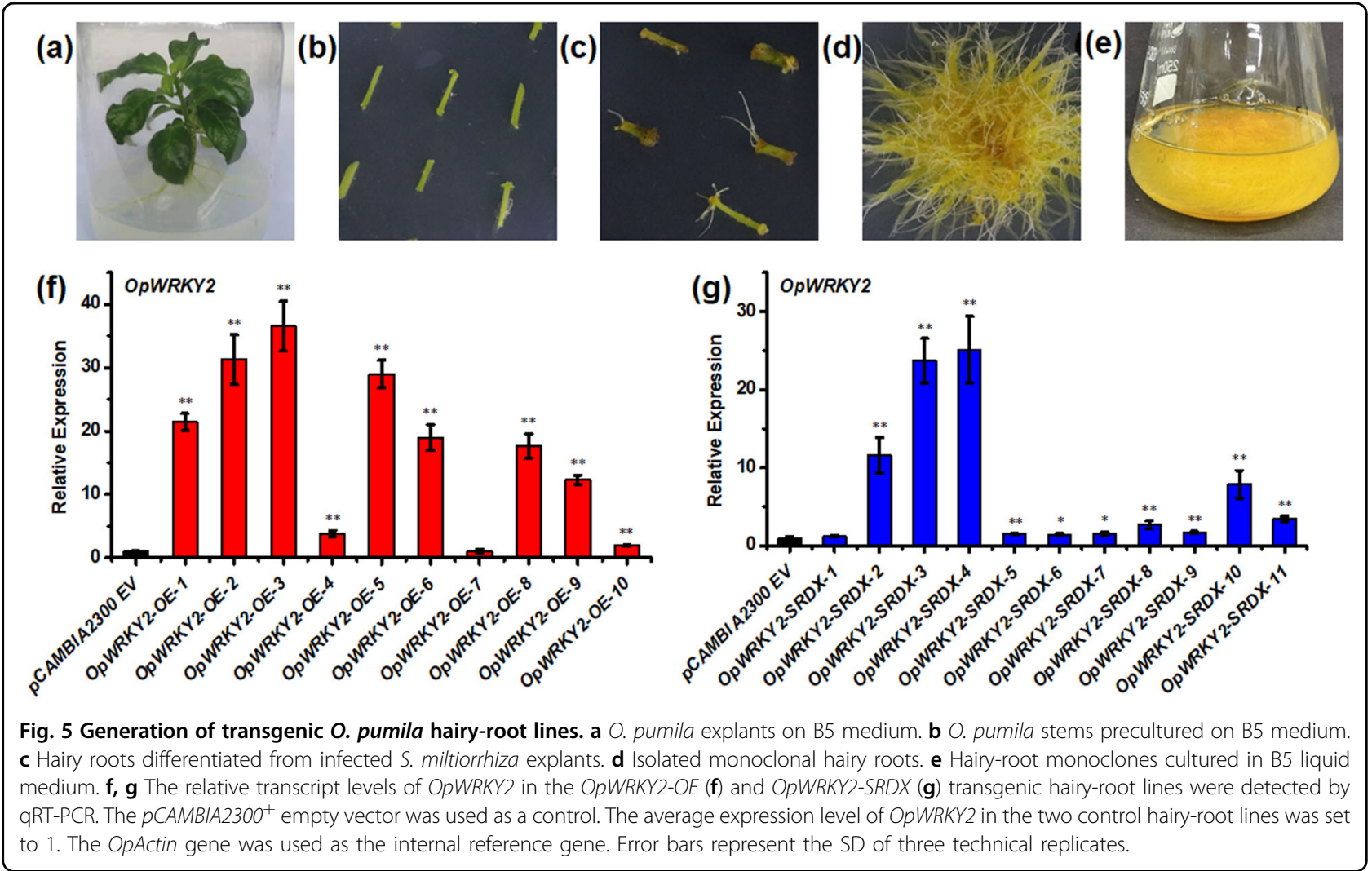

analysis of camptothecin levels in OpWRKY2-OE lines showed a 1.7-3.4-fold increase compared to the control (Fig. 6c), indicating that overexpression of OpWRKY2 is effective in promoting the accumulation of camptothecin in O. pumila. Accordingly, the average content of camptothecin in the OpWRKY2-SRDX lines decreased slightly in comparison to the control lines (Fig. 6c). The levels of tryptamine, a key intermediate in the biosynthesis of camptothecin, increased 1.83-fold in OpWRKY2-overexpressing lines and decreased by approximately half in SRDX lines (Fig. 6d). In contrast, the levels of loganin and secologanin, which are pathway intermediates in the iridoid branch of camptothecin biosynthesis, showed high variability and no obvious trend in the OpWRKY2-OE and $O p W R K Y 2-S R D X$ lines (Fig. S4). Altogether, these results suggest a positive regulatory role of OpWRKY2 in camptothecin biosynthesis.

\section{OpWRKY2 activates the transcription of OpTDC in vitro and in vivo}

To further investigate the putative role of OpWRKY2 in the regulation of $O p T D C$, the $3090 \mathrm{bp} O p T D C$ promoter region was analyzed for potential binding sites of WRKY TFs (Fig. 7a). Indeed, a conserved W-box (TTGACT) was found in the analyzed $O p T D C$ promoter region $(-2561$ bp to -2556 bp relative to the ATG). Next, the ability of OpWRKY2 to bind and activate the OpTDC promoter was analyzed in vivo and in vitro. First, the binding affinity of OpWRKY2 to the OpTDC promoter was determined by an EMSA. A 14 bp probe $(-2565$ to -2552 relative to the ATG) containing the native W-box element was used for EMSA experiments. As shown in Fig. 7b, coincubation of recombinant OpWRKY2 with the native W-box probe resulted in DNA mobility shifts, while coincubation with the control protein did not alter DNA separation. To further corroborate the binding affinity of OpWRKY2 to the W-box element in the OpTDC promoter, yeast onehybrid assays were performed. OpWRKY2 fused to the yeast GAL4 activation domain served as prey, and three tandem repeats of the W-box-containing region (CTTCAGTCAAGGCC) of the OpTDC promoter ( -2565 to -2552 relative to the ATG) served as bait. The mutant-W-box and an empty vector without a W-box served as the control. As shown in Fig. 7d, OpWRKY2GAL4 was able to activate the $p O p T D C$ - $W$-box-driven $L a c Z$ reporter in the $\mathrm{Y} 1 \mathrm{H}$ system, supporting the results of the EMSA experiments (Fig. 7d).

To confirm the activation effect of OpWRKY2 on $p O p T D C$ transcription, Dual-LUC assays were carried out in $N$. benthamiana leaves. The reporter constructs were obtained by inserting the $3090 \mathrm{bp}$ native promoter of $O p T D C$ and a mutated version with point mutations in the W-box element into the $p$ GreenII0800-LUC vector. OpWRKY2-YFP was driven by the $35 \mathrm{~S}$ promoter, and the 


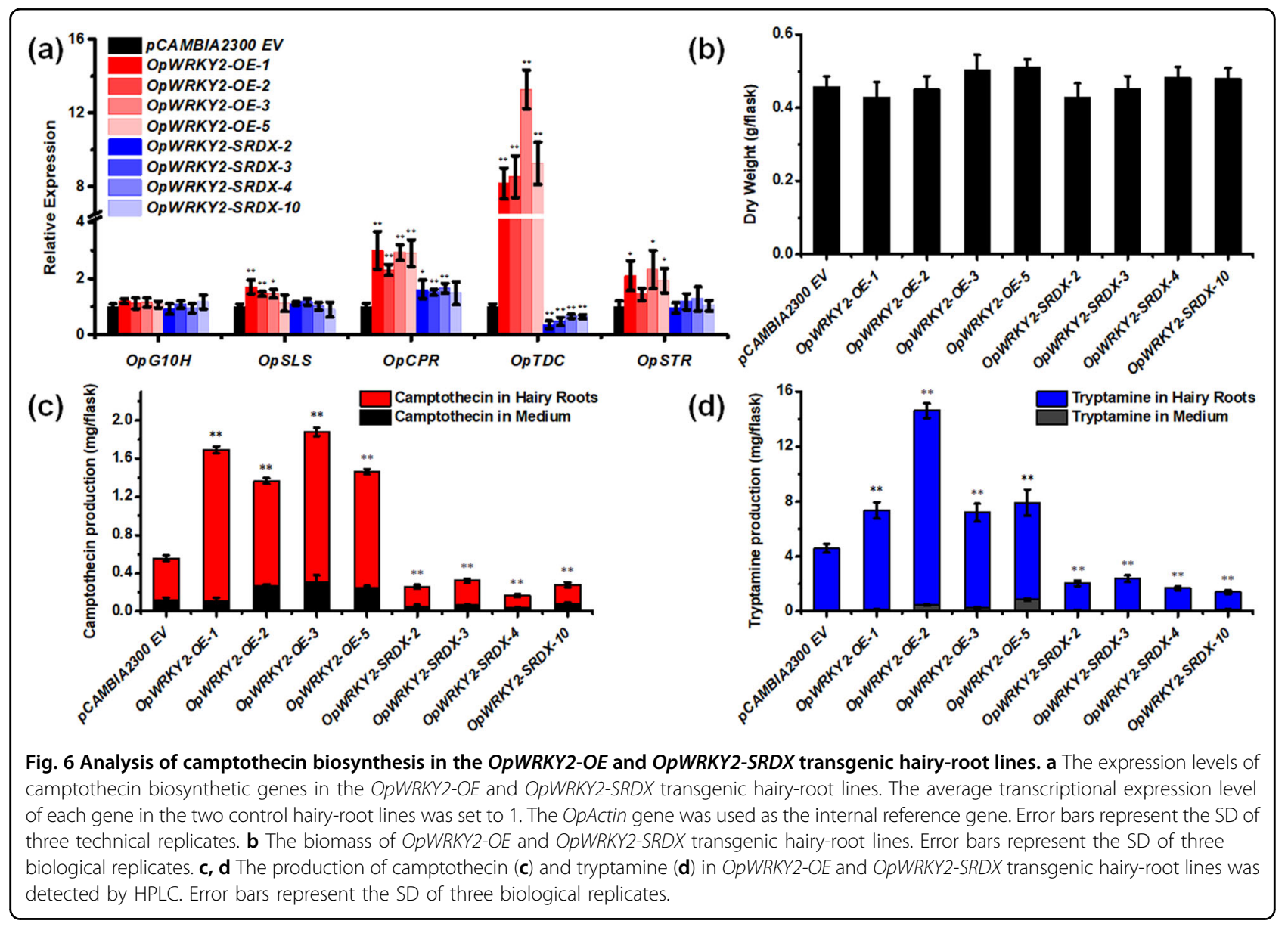

$p H B-Y F P$ construct without $O p W R K Y 2$ was used as the negative control. Compared with the $p H B-Y F P$ control group, the activation ability in OpWRKY2-pOpTDC coinfiltration was significantly increased (Fig. 7c). In contrast, the reporter gene was not activated in the mutated promoter constructs (Fig. 7c). Taken together, these results suggest that OpWRKY2 positively regulates camptothecin biosynthesis by binding a W-box element in the $O p T D C$ promoter and transcriptionally activating this key pathway enzyme-encoding gene.

\section{Discussion}

\section{Camptothecin biosynthesis in 0 . pumila hairy roots is dynamic}

Camptothecin is an important anticancer drug produced in a variety of unrelated plants. The market supply of camptothecin is currently provided by extraction from woody plants. O. pumila, a perennial herb, has recently been established as an alternative source of camptothecin. The hairy-root transformation system of $O$. pumila has been optimized for high camptothecin production and has been proposed as a major source of camptothecin for pharmaceutical markets ${ }^{7}$. The hairy-root system has a variety of features, such as rapid growth, elicitation ability, and free hormones ${ }^{33,34}$. To further optimize camptothecin production in O. pumila hairy roots, it is important to better understand the regulatory mechanism associated with camptothecin biosynthesis. However, there has been no research carried out on the dynamic changes in camptothecin biosynthesis over the cultivation period of hairy roots.

In this study, the biomass of $O$. pumila hairy roots and the total yield of camptothecin gradually increased and stabilized after 50 days. Therefore, camptothecin accumulation in O. pumila hairy roots reached a peak at 40 days and then began to decline, whereas the levels of secreted camptothecin increased throughout the analyzed time period and represented $40 \%$ of the total camptothecin in the culture at 60 days. In accordance with the levels of camptothecin production in hairy roots, the expression levels of camptothecin biosynthetic genes rapidly increased for the first 40 days of cultivation and then decreased to almost no expression until day 60 . The most drastic changes in expression among the camptothecin biosynthetic genes were observed for $O p T D C$ and $O p S T R$, with up to 5.32-fold upregulation of $O p T D C$ at 40 days. $O p T D C$ and OpSTR are central biosynthesis genes involved in the camptothecin pathway. OpTDC is considered a ratelimiting factor in CPT biosynthesis, and suppression of 

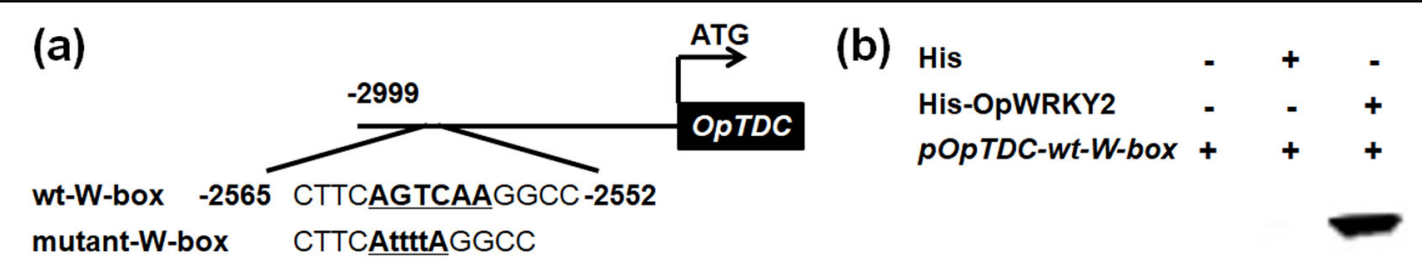

(c)
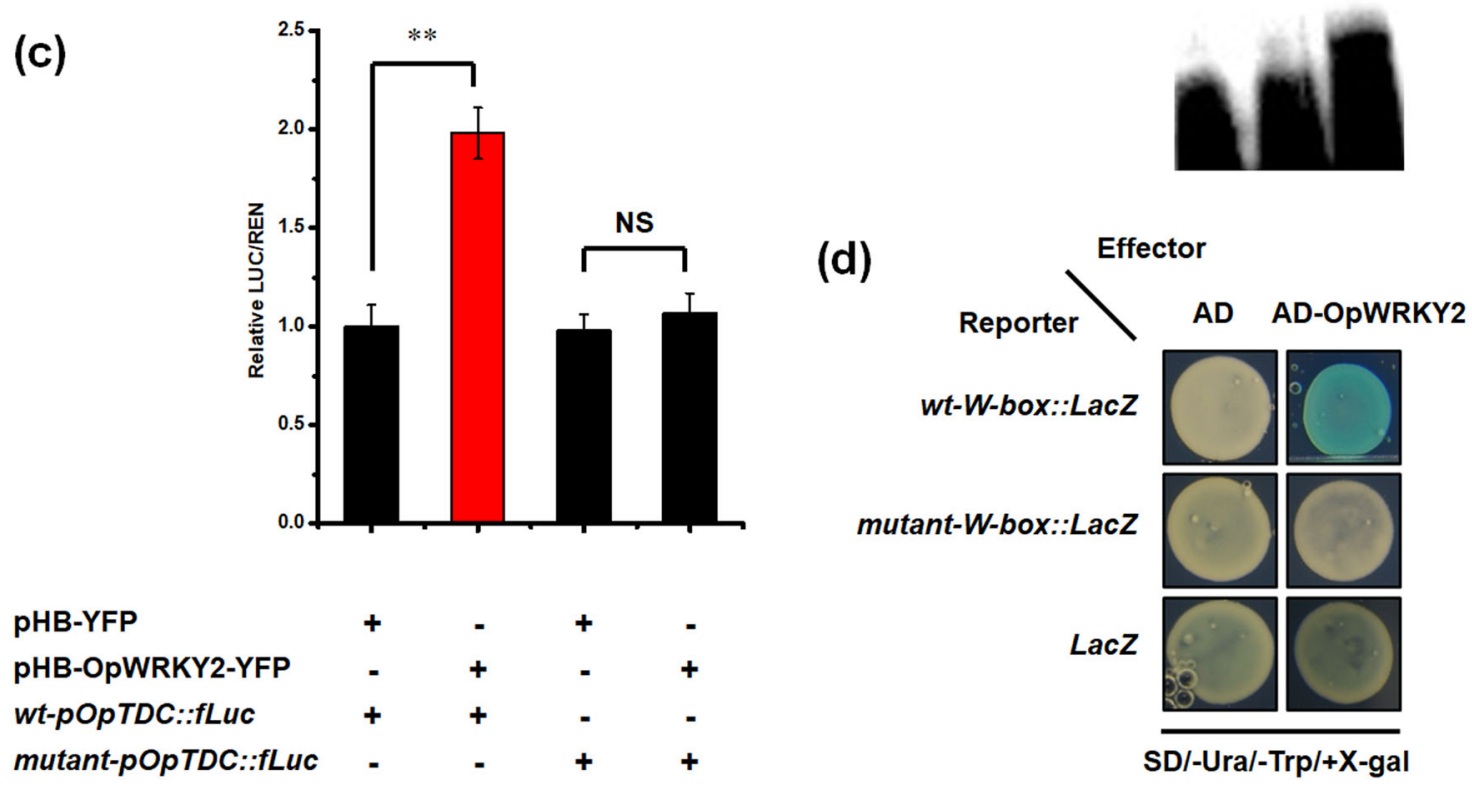

Fig. 7 OpWRKY2 binds and activates the promoter of OpTDC in vitro and in vivo. a Diagram of the OpTDC promoter fragment and the sequence of a subfragment containing a W-box motif or mutant W-box motif. b Electrophoretic mobility shift assay (EMSA) indicating that OpWRKY2 binds to the W-box in the OPTDC promoter. pCold-HIS protein without OpWRKY2 was used as the control. c Dual-luciferase (Dual-LUC) assays showed the activation effect of OpWRKY2 on the OPTDC promoter. The OpTDC promoter and OpTDC promoter containing a mutant W-box motif were fused to the firefly luciferase reporter gene, and the promoter activity was determined by a transient Dual-LUC assay in N. benthamiana. The relative LUC activity was normalized to that of the reference Renilla (REN) luciferase. Error bars indicate the SD $(n=3)$. Student's $t$-test: ${ }^{* *} P<0.01$; NS no significance. $\mathbf{d}$ Yeast one-hybrid $(\mathrm{Y} 1 \mathrm{H})$ assay indicating that OpWRKY2 binds to the W-box in the OpTDC promoter. Yeast cells transformed with different combinations of constructs were grown on SD/-Ura/-Trp/+X-gal medium. Pictures were taken after 4 days of incubation at $30^{\circ} \mathrm{C}$. Y1 $\mathrm{H}$ assays were repeated three times.

$O p T D C$ activity results in decreased production of camptothecin in O. pumila hairy roots ${ }^{11}$. Introduction of OpSTR into O. pumila hairy roots led to a significant increase in camptothecin production compared to that in a control line ${ }^{8}$. In addition, co-overexpression of STR and $\mathrm{G1OH}$ from C. roseus resulted in CPT biosynthesis in O. pumila hairy roots ${ }^{7}$. These analyses show that camptothecin biosynthesis in O. pumila hairy roots changes over time. They further highlight that separate measurements of camptothecin concentration in hairy roots and liquid media are important for understanding camptothecin accumulation in O. pumila.

\section{OpWRKY2 regulates camptothecin biosynthesis by directly activating OpTDC}

By establishing a correlation between camptothecin production and the expression of the associated biosynthesis genes, a platform for the rapid identification of candidate transcription factors was developed. Here, the platform was focused on the WRKY TF family, one of the largest TF families involved in plant growth and development, including seed development, seed dormancy and germination, biotic stress, abiotic stress, development, senescence, and secondary metabolism ${ }^{13,35}$. In plants, WRKY transcription factors are divided into three groups based on the number of WRKY domains and the structure of the zinc fingers ${ }^{12,13}$. The WRKY group II proteins are further split into five distinct subgroups (IIa-e) according to their WRKY domains ${ }^{12,13}$. Recently, a number of WRKY TFs of different groups have been implicated in the regulation of plant secondary metabolites such as terpenoids, phenolic compounds, and alkaloids. In Solanum lycopersicum, SIWRKY73 (IIb) was shown to be involved in the activation of three monoterpene synthase genes $^{36}$. In $A$. annua, the glandular trichome-specific WRKY transcription factor AaGSW1 (IIc) was shown to 
positively regulate $A a C Y P 71 A V 1$ and $A a O R A$ expression by directly binding to $\mathrm{W}$-box motifs in their promoters and acting as a positive regulator in artemisinin and dihydroartemisinin production ${ }^{25}$. Moreover, AaWRKY1 (III) in $A$. апnиa was reported to promote the transcription of AaADS, AaCYP71AV1, and AaDBR2 and positively regulate artemisinin biosynthesis ${ }^{17}$. GaWRKY1 (IIa) in Gossypium arboretum was reported to regulate the production of sesquiterpenoid gossypol by activating the $(+)$ - $\delta$-cadinene synthase (CAD1) gene ${ }^{37}$. In $S$. miltiorrhiza, both SmWRKY1 (III) and SmWRKY2 (I) were shown to positively regulate tanshinone biosynthesis by binding to a W-box in the $S m D X R$ promoter and activating the central pathway gene $\operatorname{SmCPS}$, respectively ${ }^{14,15}$. In Taxus chinensis, TcWRKY1 (IIa) was reported to regulate the biosynthesis of the diterpenoid anticancer drug $\mathrm{Taxol}^{38}$. In W. somnifera, WsWRKY1 (III) was shown to regulate the biosynthesis of triterpenoids by binding to W-box elements in the promoters of WSSQS and $W_{S S Q E}{ }^{18}$. In Arabidopsis thaliana, AtWRKY23 (IIc) and AtWRKY44 (I) were reported to regulate the production of flavanols and proanthocyanin ${ }^{39,40}$. In addition, a positive regulatory role of CrWRKY1 (III) in CrTDC activity and tryptamine accumulation in transgenic C. roseus hairy roots was identified ${ }^{16}$. In Coptis japonica, CjWRKY1 (IIc) was shown to govern the expression of berberine biosynthesis ${ }^{41}$. In O. pumila, two WRKY TFs with regulatory roles in camptothecin biosynthesis have been identified. A negative regulatory role in $O p C P R$ expression and camptothecin biosynthesis was reported for OpWRKY1 (III), and a slightly positive effect on camptothecin production was shown for OpWRKY3 (III) ${ }^{5,19}$. However, a core WRKY TF directly involved in the regulation of camptothecin biosynthesis has not yet been discovered.

Here, all putative WRKY transcription factor genes of O. pumila were identified, and their pattern of coexpression with the camptothecin pathway genes was analyzed. A single TF gene, OpWRKY2, showed strong transcriptional overlap with the camptothecin pathway. Overexpression of functional OpWRKY2 resulted in increased camptothecin levels, and silencing of $O p W R K Y 2$ correlated with decreased camptothecin levels. These results suggested a positive regulatory role of $O p W R K Y 2$ in the camptothecin biosynthetic pathway. By detecting the expression of key enzymes of the biosynthetic pathway of camptothecin in transgenic hairy roots, it was shown that the expression of $O P T D C$ was significantly upregulated in $O p W R K Y 2-O E$ hairy-root lines. Accordingly, the expression of $O P T D C$ was significantly decreased in $O p W R K Y 2$-silenced hairy-root lines. In $O$. pumila, OpTDC catalyzes the decarboxylation of tryptophan to form tryptamine, which is a key enzyme in the upstream pathway of terpenoid indole alkaloid biosynthesis $^{10}$. EMSA, Y1H, and Dual-LUC assays showed that
OpWRKY2 could bind and activate a W-box in the promoter of $O p T D C$ in vitro and in vivo. In addition, the expression level of $O P C P R$ was increased slightly in $O p W R K Y 2-O E$ hairy-root lines compared to the control. Thus, the levels of loganin and secologanin, which are pathway intermediates in the iridoid branch of camptothecin biosynthesis, also showed a slight increase when OpWRKY2 was overexpressed. Altogether, these results suggested that OpWRKY2 positively regulates camptothecin biosynthesis by binding to the W-box of $p O p T D C$ and activating the expression of $O p T D C$ (Fig. 8). In addition to the level of $O p W R K Y 2$, the expression levels of OpWRKY3, OpWRKY4, OpWRKY5, OpWRKY13, OpWRKY15, OpWRKY16, OpWRKY21, OpWRKY28, and $O p W R K Y 34$ also showed a gradual increase before day 50 . Furthermore, the expression of some $O p W R K Y s$, such as OpWRKY1, OpWRKY6, and OpWRKY9, gradually decreased and might negatively regulate camptothecin biosynthesis. Indeed, OpWRKY1 has been reported to negatively regulate the biosynthesis of camptothecin, while $O p W R K Y 3$ positively regulates the biosynthesis of camptothecin $^{5,19}$. Therefore, it is effective to screen candidate regulatory genes based on their expression levels in different growth stages, and it is necessary to continue to study the regulatory functions and mechanisms of other possible WRKY transcription factors in camptothecin biosynthesis.

Previously, a set of TFs has been implicated in the regulation of camptothecin production in O. pumila. Out of five ERF TF family genes (OpERF1-5) that had been isolated and characterized from $O$. pumila hairy roots, one TF-encoding gene, OpERF2, has been shown to play a positive role in regulating the iridoid biosynthesis branch of camptothecin biosynthesis ${ }^{20}$. Moreover, the introduction of $O p M Y B 1$ into $O$. pumila hairy roots reduced camptothecin production and downregulated the expression level of $O p T D C$, suggesting a repressive function of $O p M Y B 1$ in camptothecin biosynthesis ${ }^{21}$. In addition, two WRKY TFs with regulatory roles in $O$. pumila camptothecin biosynthesis have been identified. In contrast to the direct positive regulatory role of OpWRKY2 in OpTDC expression shown here, OpWRKY1 had been implicated in the suppression of camptothecin biosynthesis by binding two W-boxes in the $O P C P R$ promoter and repressing the expression of $O P C P R$, while a slight involvement in camptothecin regulation has been shown for OpWRKY3, which binds via one W-box in the $O p C P R$ promoter (Fig. 8) ${ }^{5,19}$. In addition, camptothecin accumulation was reported to be significantly increased by plant hormones such as salicylic acid and jasmonic acid in CPT-producing plants and cell cultures $^{42-44}$. For example, camptothecin production increased under jasmonic acid treatment in cell cultures of C. acuminata ${ }^{42}$. Moreover, exogenous salicylic acid in 
(a)
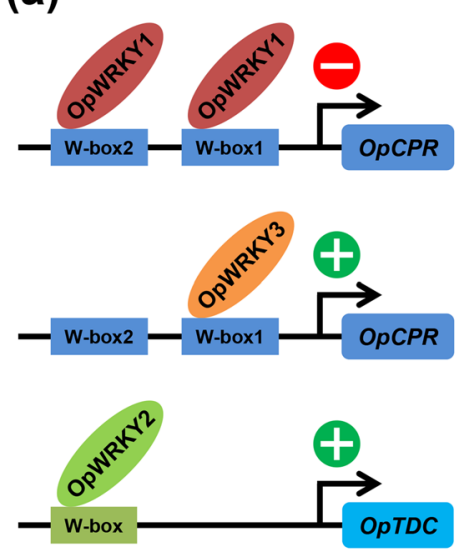

(b)

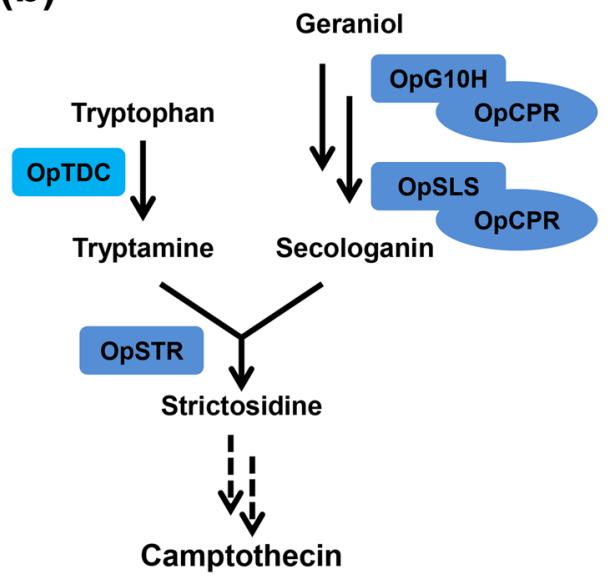

Fig. 8 Regulatory model of camptothecin biosynthesis by OpWRKYs. a OpWRKY2 positively regulates camptothecin biosynthesis by binding a W-box in the OPTDC promoter and activating the expression of OpTDC. OpWRKY1 negatively regulates camptothecin biosynthesis by binding two Wboxes in the OPCPR promoter, while OpWRKY3 positively regulates camptothecin biosynthesis by binding one W-box in the OpCPR promoter. b Camptothecin biosynthetic pathway in O. pumila. Tryptophan decarboxylase (OpTDC) catalyzes the conversion of tryptophan to tryptamine. Dashed lines represent interactions that may be indirect.

C. acuminata seedlings promoted camptothecin biosynthesis $^{43}$. In another CPT-producing plant, Ophiorrhiza mungos, cell suspension culture achieved significantly high camptothecin production with jasmonic acid elicitation $^{44}$. Therefore, camptothecin accumulation is induced by hormone treatments. However, the effects of phytohormones on camptothecin accumulation in Ophiorrhiza pumila plants have not been reported previously, and we will further explore the effect of different plant hormones on the biosynthesis and accumulation of camptothecin in O. pumila.

\section{Conclusions}

Camptothecin, a monoterpene indole alkaloid, is widely used in the treatment of cancer. This study found that camptothecin accumulation in O. pumila hairy roots is positively correlated with increasing culture time. Furthermore, the expression of OpWRKY2 is correlated with camptothecin biosynthesis and induced by various phytohormones. The expression level of the camptothecin biosynthesis gene OPTDC was increased in the $O P W R K Y 2-O E$ hairy-root lines and significantly downregulated in the OPWRKY2-SRDX hairy-root lines. Metabolite analysis in transgenic hairy roots found that $O p W R K Y 2$ positively regulates the biosynthesis of camptothecin and tryptamine. EMSA, Y1H, and Dual-LUC assays showed that OpWRKY2 binds and activates the promoter of $O p T D C$ in vitro and in vivo. Taken together, the present findings illustrate that OpWRKY2 acts as a positive regulator in camptothecin biosynthesis and provide a feasible strategy for increasing camptothecin levels by functional WRKY proteins in O. pumila.

\section{Acknowledgements}

This work was funded by the National Key Research and Development Program of China (2018YFC1706203), National Natural Science Foundation of China (31571735, 82073963, 81522049, 82003889), Zhejiang Provincial Ten Thousands Program for Leading Talents of Science and Technology Innovation (2018R52050), Zhejiang Provincial Program for the Cultivation of High-level Innovative Health Talents, Shanghai Science and Technology Committee Project (17JC1404300), Zhejiang Provincial Natural Science Foundation of China (LY2OH280008, LQ21H280004), and Zhejiang Chinese Medical University Research Foundation (2020ZR15).

\section{Author details}

${ }^{1}$ Laboratory of Medicinal Plant Biotechnology, College of Pharmacy, Zhejiang Chinese Medical University, 310053 Hangzhou, China. ${ }^{2}$ Institute of Plant Biotechnology, School of Life Sciences, Shanghai Normal University, 200234 Shanghai, China. ${ }^{3}$ The Milner Centre for Evolution, Department of Biology and Biochemistry, University of Bath, Claverton Down, Bath BA2 7AY, UK

\section{Author contributions}

X.H., C.X., and G.K. conceived and designed the project. X.H., C.X., Q.R., X.Z., and C.W. performed the experiments. X.H., C.X., B.H., and J.Q. analyzed the data. X.H., W.Z., and H.N. wrote the manuscript. X.H., H.N., and G.K. revised the manuscript. All authors read and approved the final manuscript.

Conflict of interest

The authors declare that they have no conflict of interest.

Supplementary Information accompanies this paper at (https:/doi.org/ 10.1038/s41438-020-00437-3).

Received: 28 July 2020 Revised: 22 September 2020 Accepted: 17 October 2020

Published online: 01 January 2021

\section{References}

1. Wall, M. E. et al. Plant antitumor agents. I. the isolation and structure of camptothecin, a novel alkaloidal leukemia and tumor inhibitor from Camptotheca acuminata. J. Am. Chem. Soc. 88, 3888-3890 (1966).

2. Staker, B. L. et al. The mechanism of topoisomerase I poisoning by a camptothecin analog. Proc. Natl Acad. Sci. USA 99, 15387-15392 (2002). 
3. Sirikantaramas, S., Yamazaki, M. \& Saito, K. Mutations in topoisomerase I as a selfresistance mechanism coevolved with the production of the anticancer alkaloid camptothecin in plants. Proc. Natl Acad. Sci. USA 105, 6782-6786 (2008).

4. Kai, G. et al. Biosynthesis and biotechnological production of anti-cancer drug camptothecin. Phytochem. Rev. 14, 525-539 (2015).

5. Wang, C. et al. Transcription factor OpWRKY3 is involved in the development and biosynthesis of camptothecin and its precursors in Ophiorrhiza pumila hairy roots. Int. J. Mol. Sci. 20, 3996 (2019).

6. Saito, K. et al. Feasible production of camptothecin by hairy root culture of Ophiorrhiza pumila. Plant Cell Rep. 20, 267-271 (2001).

7. Cui, L. et al. Co-overexpression of geraniol-10-hydroxylase and strictosidine synthase improves anti-cancer drug camptothecin accumulation in Ophiorrhiza pumila. Sci. Rep. 5, 8227 (2015).

8. Shi, M. et al. Targeted metabolic engineering of committed steps improves anti-cancer drug camptothecin production in Ophiorrhiza pumila hairy roots. Ind. Crop Prod. 148, 112277 (2020).

9. Yamazaki, Y. et al. Biosynthesis of camptothecin. In silico and in vivo tracer study from [1-13C] glucose. Plant Physiol. 134, 161-170 (2004).

10. Yamazaki, Y., Sudo, H., Yamazaki, M., Aimi, N. \& Saito, K. Camptothecin biosynthetic genes in hairy roots of Ophiorrhiza pumila: cloning, characterization and differential expression in tissues and by stress compounds. Plant Cell Physiol. 44, 395-403 (2003).

11. Asano, T. et al. Suppression of camptothecin biosynthetic genes results in metabolic modification of secondary products in hairy roots of Ophiorrhiza pumila. Phytochemistry 91, 128-139 (2013).

12. Eulgem, T., Rushton, P. J., Robatzek, S. \& Somssich, I. E. The WRKY superfamily of plant transcription factors. Trends Plant Sci. 5, 199-206 (2000).

13. Rushton, P. J., Somssich, I. E., Ringler, P. \& Shen, Q. J. WRKY transcription factors. Trends Plant Sci. 15, 247-258 (2010).

14. Deng, C. et al. Tanshinone production could be increased by the expression of SmWRKY2 in Salvia miltiorrhiza hairy roots. Plant Sci. 284, 1-8 (2019).

15. Cao, W. et al. Transcription factor SmWRKY1 positively promotes the biosynthesis of tanshinones in Salvia miltiorrhiza. Front. Plant Sci. 9, 554 (2018).

16. Suttipanta, N. et al. The transcription factor CrWRKY1 positively regulates the terpenoid indole alkaloid biosynthesis in Catharanthus roseus. Plant Physiol. 157, 2081-2093 (2011).

17. Ma, D. et al. Isolation and characterization of AaWRKY1, an Artemisia annua transcription factor that regulates the amorpha-4,11-diene synthase gene, a key gene of artemisinin biosynthesis. Plant Cell Physiol. 50, 2146-2161 (2009).

18. Singh, A. K. et al. A WRKY transcription factor from Withania somnifera regulates triterpenoid withanolide accumulation and biotic stress tolerance through modulation of phytosterol and defense pathways. N. Phytol. 215, 1115-1131 (2017).

19. $\mathrm{Xu}, \mathrm{M}$. et al. WRKY transcription factor OpWRKY1 acts a negative regulator of camptothecin biosynthesis in Ophiorrhiza pumila hairy roots. Plant Cell Tiss. Org. Cult. 142, 69-78 (2020).

20. Udomsom, N. et al. Function of AP2/ERF transcription factors involved in the regulation of specialized metabolism in Ophiorrhiza pumila revealed by transcriptomics and metabolomics. Front. Plant Sci. 7, 1861 (2016).

21. Rohani, E. R. et al. An MYB transcription factor regulating specialized metabolisms in Ophiorrhiza pumila. Plant. Biotechnol 33, 1-9 (2016).

22. Chang, C., Liu, Z., Wang, Y., Tang, Z. \& Yu, F. A bZIP transcription factor, CaLMF, mediated light-regulated camptothecin biosynthesis in Camptotheca acuminata. Tree Physiol. 39, 372-380 (2018).

23. Hao, $X$. et al. Tanshinone and salvianolic acid biosynthesis are regulated by SmMYB98 in Salvia miltiorrhiza hairy roots. J. Adv. Res. 23, 1-12 (2020).

24. Hao, X. et al. Effects of methyl jasmonate and salicylic acid on tanshinone production and biosynthetic gene expression in transgenic Salvia miltiorrhiza hairy roots. Biotechnol. Appl. Biochem. 62, 24-31 (2015).
25. Chen, M. et al. GLANDULAR TRICHOME-SPECIFIC WRKY 1 promotes artemisinin biosynthesis in Artemisia annua. N. Phytol. 214, 304-316 (2017).

26. Thompson, J. D., Higgins, D. G. \& Gibson, T. J. CLUSTAL W: improving the sensitivity of progressive multiple sequence alignment through sequence weighting, position-specific gap penalties and weight matrix choice. Nucleic Acids Res. 22, 4673-4680 (1994).

27. Tamura, K. et al. MEGA5: molecular evolutionary genetics analysis using maximum likelihood, evolutionary distance, and maximum parsimony methods. Mol. Biol. Evol. 28, 2731-2739 (2011).

28. Sun, M. et al. The biosynthesis of phenolic acids is positively regulated by the JA-responsive transcription factor ERF115 in Salvia miltiorrhiza. J. Exp. Bot. 70, 243-254 (2019).

29. Hiratsu, K., Matsui, K., Koyama, T. \& Ohme-Takagi, M. Dominant repression of target genes by chimeric repressors that include the EAR motif, a repression domain, in Arabidopsis. Plant J. 34, 733-739 (2003).

30. Hao, X. et al. Transcriptome analysis of genes associated with the artemisinin biosynthesis by jasmonic acid treatment under the light in Artemisia annua. Front. Plant Sci. 8, 971 (2017).

31. Hao, X. et al. Light-induced artemisinin biosynthesis is regulated by the bZIP transcription factor AaHY5 in Artemisia annua. Plant Cell Physiol. 60, 1747-1760 (2019).

32. Huang, Q. et al. The AP2/ERF transcription factor SmERF1L1 regulates the biosynthesis of tanshinones and phenolic acids in Salvia miltiorrhiza. Food Chem. 274, 368-375 (2019).

33. Shi, M., Huang, F., Deng, C., Wang, Y. \& Kai, G. Bioactivities, biosynthesis and biotechnological production of phenolic acids in Salvia miltiorrhiza. Crit. Rev. Food Sci. 59, 953-964 (2019).

34. Sudo, H., Yamakawa, T., Yamazaki, M., Aimi, N. \& Saito, K. Bioreactor production of camptothecin by hairy root cultures of Ophiorrhiza pumila. Biotechnol. Lett. 24, 359-363 (2002).

35. Phukan, U. J., Jeena, G. S. \& Shukla, R. K. WRKY transcription factors: molecular regulation and stress responses in plants. Front. Plant Sci. 7, 1861 (2016).

36. Spyropoulou, E. A., Haring, M. A. \& Schuurink, R. C. RNA sequencing on Solanum lycopersicum trichomes identifies transcription factors that activate terpene synthase promoters. BMC Genomics 15, 402 (2014).

37. Xu, Y., Wang, J., Wang, S., Wang, J. \& Chen, X. Characterization of GaWRKY1, a cotton transcription factor that regulates the sesquiterpene synthase gene (+)- $\delta$-cadinene synthase-A. Plant Physiol. 135, 507-515 (2004).

38. Li, S., Zhang, P., Zhang, M., Fu, C. \& Yu, L. Functional analysis of a WRKY transcription factor involved in transcriptional activation of the DBAT gene in Taxus chinensis. Plant Biol. 15, 19-26 (2013).

39. Johnson, C. S., Kolevski, B. \& Smyth, D. R. TRANSPARENT TESTA GLABRA2, a trichome and seed coat development gene of Arabidopsis, encodes a WRKY transcription factor. Plant Cell 14, 1359-1375 (2002).

40. Grunewald, W. et al. Transcription factor WRKY23 assists auxin distribution patterns during Arabidopsis root development through local control on flavonol biosynthesis. Proc. Natl Acad. Sci. USA 109, 1554-1559 (2012).

41. Kato, N. et al. Identification of a WRKY protein as a transcriptional regulator of benzylisoquinoline alkaloid biosynthesis in Coptis japonica. Plant Cell Physiol. 48, 8-18 (2007).

42. Song, S. H. \& Byun, S. Y. Elicitation of camptothecin production in cell cultures of Camptotheca acuminata. Biotechnol. Bioprocess Eng. 3, 91-95 (1998).

43. Liu, Z. et al. Effects of exogenous salicylic acid on accumulation of camptothecin and gene expression in Camptotheca acuminata. Can. J. Forest. Res. 49, 104-110 (2019).

44. Deepthi, S. \& Satheeshkumar, K. Cell line selection combined with jasmonic acid elicitation enhance camptothecin production in cell suspension cultures of Ophiorrhiza mungos L. Appl. Microbiol. Biotechnol. 101, 545-558 (2017). 